ISSN 1112-9867

http://www.jfas.info

\title{
NANOTECHNOLOGY AND SUPERCRITICAL FLUIDS
}

A. Bagheri Hamidreza ${ }^{1,2, *}$, B. Soltani Reza ${ }^{1,3}$, C. Shahmirzaei Mahdi ${ }^{4}$ D. Yahyanejad Mahdi ${ }^{5}$ E. Fijani Fatemeh ${ }^{6}$ and F. Hashemipour Hassan ${ }^{1,}$

${ }^{1}$ Department of Chemical Engineering, Faculty of Engineering, Shahid Bahonar University of Kerman, Kerman, Iran

${ }^{2}$ Young Researchers Society, Shahid Bahonar University of Kerman, Kerman, Iran.

${ }^{3}$ Specialist of Barez Industrial Group, Kerman, Iran

${ }^{4}$ Department of Metallurgy Engineering, Payame Noor University of Kerman, Kerman, Iran.

${ }^{5}$ Department of Chemical Engineering, Payame Noor University of Kerman, Kerman, Iran.

${ }^{6}$ Department of Chemical Engineering, Yasouj University, Yasouj, Iran

\begin{abstract}
Supercritical fluid (SCF) technology has become an important tool of materials processing in the last two decades. Supercritical $\mathrm{CO}_{2}$ and $\mathrm{H}_{2} \mathrm{O}$ are extensively being used in the preparation of a great variety of nanomaterials. The interest in the preparation and application of nanometer size materials is increasing since they can exhibit properties of great industrial interest. Several techniques have been proposed to produce nanomaterials using supercritical fluids. These processes, taking advantage of the specific properties of supercritical fluids, are generally flexible, more simplified and with a reduced enviromental impact. The result is that nanomaterials with potentially better performances have been obtained. We propose a critical review of the supercritical based techniques applied to the production of nanoparticles materials.
\end{abstract}

Keywords: Supercritical fluids; Nanoparticles; SCF technology; RESS; SAS.

Author Correspondence, e-mail: hamidbagheri101@gmail.com

doi: http://dx.doi.org/10.4314/jfas.v8i2s.138 


\section{INTRODUCTION}

There is a great interest in the preparation and application of nanometer size materials since they can exhibit new properties of industrial interest. Which are the matter properties that can show dramatic changes at nanoscale range? Mainly the properties related to the ratio between surface and volume: at nanoscale, surface properties become relevant with respect to volume properties. For example, surface molecules can impart high hardness to metals and higher energy to propellants and explosives; electronic devices and pharmaceuticals with improved performance can also be produced. Different interpretations of the dimensions that set the boundary between normal materials and nanomaterials have been proposed. In many researches, assume that a nanoproduct should have at least one dimension smaller than $200 \mathrm{~nm}$; though, more restrictive definitions have been proposed that set the upper limit at $100 \mathrm{~nm}$ [1]. Nanoparticles, nanofilms and nanowires are nanometric along three, two and one dimension, respectively. However, from 1990s the scientists are popularly using the term nanotechnology to refer designing, characterization, production and application of structures, devices and systems that exist in between those of atoms and bulk materials with at least one dimension in the nanometer range $(1 \mathrm{~nm}=$ one thousand millionth of a meter, $10-9 \mathrm{~m})$. The size range of nanotechnology is often delimited to $100 \mathrm{~nm}$ down to the molecular level down up to $0.2 \mathrm{~nm}$ because; this is where materials have significantly different properties. The term nanomaterials covers materials in one dimension (thin films), two-dimension (nanofibers, nanowires, nanotubes, etc.), and three-dimension (nanoparticles/nanopowders, nanocapsules, fullerenes, dendrimers, molecular electronics, quantum dots, nanostructured materials, nanoporous materials, etc.) [2-4]. The nanomaterials are known for their unique mechanical, chemical, physical, thermal, electrical, optical, magnetic, biological and also specific surface area properties, which in turn define them as nanostructures, nanoelectronics, nanophotonics, nanobiomaterials, nanobioactivators, nanobiolabels, etc. In the last one decade a large variety of nanomaterials and devices with new capabilities have been generated employing nanoparticles based on metals, metal oxides, ceramics (both oxide and non-oxide), silicates, organics, polymers, etc. One of the most important properties of materials in nano-size regime is the changing physical properties [3-5]. During 1980s, the concept of size quantization was 
formulated for semiconductors. Since then considerable progress has been achieved in this field to understand the size related properties in materials. As the size of semiconductor nanoparticles decreases, a threshold value characteristic of each type of semiconductors is reached. At smaller nanoparticle sizes, the energy gap (band gap) increases, and the optical spectrum is shifted toward the short-wavelength region.

A major challenge in the nanomaterials science is the accurate control of the size and shape, which in turn is directly linked with the nanomaterials processing method. On the whole there are only two approaches known for the nanomaterial fabrication: bottom-up and top-down methods [2]. The bottom-up approach deals with the controlled assembly of atomic and molecular aggregates into larger systems (e.g. clusters, organic lattices, supermolecular structures and synthesized macromolecules. This covers the popular synthesis methods like: chemical synthesis, self-assembly, positional assembly, leading to the formation of particles, molecules, cosmetics, fuel additives, crystals, films, tubes, displays, atomic or molecular devices $[1,6]$. Whereas the top-down approach deals with the reduction in structure sizes of microscopic elements to the nanometer scale by applying specific machining and etching techniques like: lithography, cutting, etching, grinding, etc., giving rise to electronic devices, chip masks, quantum well lasers, computer chips, MEMS, precision engineered surfaces, high quality optical mirrors [4]. In those early days, nanotechnology referred to the possibility of synthesis via direct manipulation of atoms and molecules for fabrication of macroscale products. In the 1990, K. Eric Drexler and M. Minsky used the word "nanotechnology" in their book "Engines of Creation: The Coming Era of Nanotechnology" [7], in which they proposed assembling machines and devices on the scale of molecules, a few nanometers wide. Later on, as nanotechnology became an accepted concept, the meaning of the word shifted to encompass technologies related to making any type of materials, structures and devices in nanometer scale. A nanometer $(\mathrm{nm})$ is one-billionth of a meter, hundred-thousandth the width of a human hair. There is a multidisciplinary convergence of science dedicated to the study of a world in such small scale. The US National Nanotechnology Initiative has described four generations of nanotechnology development. The first era is a design of passive nanostructures and materials to perform just one task like nanostructured metals, aerosol. The second phase introduced 
active nanostructures for multitasking, for example, actuators, drug delivery devices and sensors. The third generation featured nanosystems with thousands of interacting components. In this era, integrated nanosystems, hierarchical systems within systems, have been developed. Nanotechnology can be referred to as a general-purpose technology, as it has significant impacts on almost all industries and all areas of society. Nanotechnology is expected to offer better built, longer-lasting, cleaner, safer and smarter products for the home, for communications, for medicine, for transportation, for agriculture and for industry in general. Chemistry and materials science and in some cases biology are integrated to create new properties of materials in nanoscale. However, engineering principles must be exploited to gain market opportunities [8].

The various processes that have been proposed to obtain nanomaterials follow two main approaches: top down and bottom up. Top-down is characterized by the production of nanoproducts departing from normal size materials; i.e., reducing the dimensions of the original material; for example, using special size reduction techniques. Bottom-up approach is related to the "synthesis" of nanosized materials, starting from the molecular scale; for example, the formation of particles by precipitation from a fluid phase.

Supercritical fluids have been studied extensively for the past two decades in attempts to gain accurate and detailed knowledge of their fundamental properties. Such knowledge is essential to the utilization and optimization of supercritical fluid technology in materials preparation and processing [9-11]. Among the most important properties of a supercritical fluid are the low and tunable densities that can be varied between those of a gas and a normal liquid and the local density effects observed in supercritical fluid solutions (most strongly associated with near-critical conditions). A supercritical fluid may be considered macroscopically homogeneous but microscopically inhomogeneous, consisting of clusters of solvent molecules and free volumes. Technically, a SCF is a gas but not a vapor. The term "gas" refers to any phase which "will conform in volume to the space available". A "vapor" is defined as "a gas whose temperature is less than the critical temperature". This was the definition proposed by Thomas Andrews in 1869 and is still generally, but not universally, accepted today. In practice, however, the terms gas and vapor are often used interchangeably [5, 9]. SCFs are materials 
above their critical temperature $\left(\mathrm{T}_{\mathrm{c}}\right)$ and critical pressure $\left(\mathrm{P}_{\mathrm{c}}\right)$. The density of an SCF is comparable to that of liquids, while its diffusivity and viscosity are comparable to those of gases, as can be seen from Table 1. In addition, the surface tension of an SCF is zero [12].

The "law of corresponding states" as introduced by van der Waals implies that compounds behave similarly under the same values of the reduced variables. This allows valuable comparison of different compounds under various conditions, but deviations can be substantial in close proximity to the critical point [13].

Table1. Characteristic magnitudes of thermophysical properties [14]

\begin{tabular}{cccc}
\hline Fluid & Density $\left(\mathbf{K g} / \mathbf{m}^{\mathbf{3}}\right)$ & Viscosity $(\mathbf{c p})$ & $\begin{array}{c}\text { Diffusion } \\
\text { coefficient }\left(\mathbf{c m}^{\mathbf{2}} / \mathbf{s}\right)\end{array}$ \\
\hline Gas & 1 & $10^{-2}$ & $10^{-1}$ \\
SCF & $300-800$ & $0.03-0.1$ & $10^{-4}$ \\
Liquid & $10^{3}$ & 1 & $10^{-5}$ \\
\hline
\end{tabular}

The properties of SCFs are frequently described as being intermediate between those of a gas and a liquid. This Janus-faced nature of SCFs arises from the fact that the gaseous and liquid phases merge together and become indistinguishable at the critical point [15-18]. The liquid-like density leads to the strong solvent power of SCFs for different solutes. The most interesting aspects of SCFs are that their physical properties are very sensitive to temperature and pressure and there exists density inhomogeneity in the critical region. In a sc solution, the density of the solvent around the solute can be much larger than that of the bulk, especially in the critical region of sc solvents, which is often called clustering [17-25]. Not all properties of SCFs are intermediate between those of gases and liquids; compressibility and heat capacity, for example, are significantly higher near the critical point than they are in liquids or gases (or even in the supercritical state further from the critical point). Although the properties of a compound may change drastically with pressure near the critical point, most of them show no discontinuity [24-28]. The changes start gradually, rather than with a sudden onset, when the conditions approach the critical point. The most widely used supercritical fluid is carbon dioxide $\left(\mathrm{CO}_{2}\right)$, that is cheap and non polluting, and whose critical parameters are simple to be obtained in an industrial apparatus. However, ammonia, alcohols, light hydrocarbons and 
water have been proposed, among the others, for nanomaterials production at supercritical conditions $[29,30]$.

\subsection{Nanoparticles generation}

Supercritical fluids (SCFs) have also been proposed as media to produce nanomaterials. Among all the nanoproducts that can be envisaged, two main areas have been explored using supercritical fluids: nanoparticles and nanostructured materials [9]. Nanoparticles cover a wide range of applications; it will be possible to produce explosives with a higher potential; i.e., approaching the ideal detonation; coloring matter with brighter colors; toners with a higher resolution; polymers and biopolymers with improved functional and structural properties. In the last one decade SCF technology has witnessed a seminal growth in its application to process a variety of materials. SCF technology replaces organic solvents in a number of chemical processes, including nanoparticle fabrication, food processing (such as decaffeination of coffee beans), chemical manufacturing, extraction, dry cleaning, semiconductor wafer cleaning, polymer processing, recycling, waste treatment, organic decomposition, particles coating, etc. [29-33]. Especially for the nanomaterial fabrication for the advanced drug delivery and for drug formulation systems, SCF technology emerges out as an alternate to most of the existing techniques. In contrast to the conventional particle formation methods like freeze-drying, spray-drying, and precipitation, where a larger particle is originally formed and then comminuted to the desired size, SCF technology involves growing the particles in a controlled fashion to attain the desired morphology. The adverse effects originating from the energy imparted to the system to bring about size reduction can thus be circumvented $[34,35]$. The particles once formed need not undergo further processing or treatment and this feature makes SCF technology amenable to produce biomolecules and other sensitive compounds in their native pure state. Using this SCF, new microencapsulation techniques have been developed in order to overcome some of the disadvantages of the conventional techniques [36-38]. A possible general classification of SCF based nanoparticles generation techniques can be proposed according to the role played by the SCF in the process. Indeed, SCFs have been proposed as solvents, solutes, anti-solvents and reaction media. There are several variants in SCF technology like static supercritical fluid process (SSF), rapid 
expansion of supercritical solutions (RESS), particles from gas-saturated solutions (PGSS), gas antisolvent process (GAS), precipitation from compressed antisolvent (PCA), aerosol solvent extraction system (ASES), supercritical antisolvent process (SAS), solution enhanced dispersion by supercritical fluids (SEDS), supercritical antisolvent process with enhanced mass transfer (SAS-EM), depressurization of an expanded liquid organic solution (DELOS), supercritical assisted atomization (SAA), hydrothermal synthesis under supercritical conditions via flow reactor (HTSSF), hydrothermal synthesis under supercritical conditions via batch reactor (HTSSB), supercritical fluids drying (SCFD), supercritical fluid extraction emulsions (SFEE), etc. Here, some of these important processes of SCF technology in the first part and the supercritical hydrothermal synthesis method in the second part have been discussed in greater detail with a focus on materials being prepared in the authors' laboratories, for the purpose of biomedical applications [11, 39 and 40].

\subsection{RESS}

The rapid expansion of supercritical solutions (RESS) consists of the saturation of the supercritical fluid with a solid substrate; then, the depressurization of the solution through a heated nozzle into a low pressure chamber produces a rapid nucleation of the substrate in form of very small particles that are collected from the gaseous stream (Fig. 1).

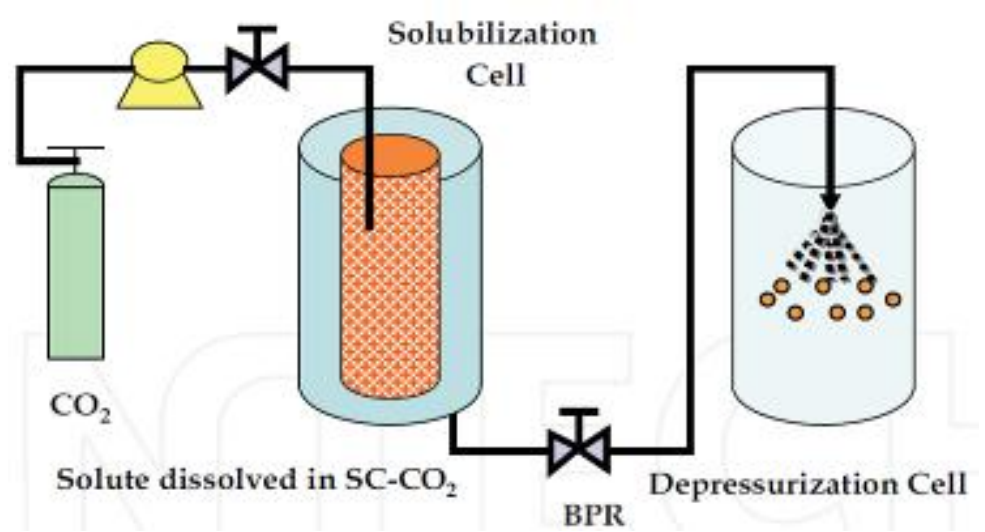

Fig.1. RESS process diagram [11]

The morphology of the resulting solid material, crystalline or amorphous, depends on the chemical structure of the material and on the RESS parameters (temperature, pressure drop, impact distance of the jet against a surface, nozzle geometry, etc.). The very fast release of the 
solute in the gaseous medium should assure the production of very small particles. This process is particularly attractive due to the absence of organic solvents [9-11]. An interesting variation of the RESS process is the rapid expansion of a supercritical solution into a liquid solvent (RESOLV) that consists of spraying the supercritical solution into a liquid. Operating in this manner, it should be possible to quench particles growth in the precipitator, thus improving the RESS process performance [41]. Moreover, by interaction among the nucleating solid particles and the compounds contained in the liquid phase, a chemical reaction step can also be added. The potential features of RESS are very interesting from the theoretical point of view; but, the results have not been particularly good in several cases. It is in many cases problematic to control the particle size of the precipitates. During the expansion, the particles coalesce in the supersonic free jet generated in the precipitation vessel and, therefore, in many cases needlelike particles have been obtained.

Sometimes, the formation of oriented needles can be explained by the presence of electrostatic charges on the surface of the particles, induced by the fast relative motion between the particles and the gas contained in the expansion chamber. RESOLV configuration has been demonstrated to be more effective in producing nanoparticles, since the liquid that receives the expanding jet, can suppress the particle growth [42]. The addition of a stabilizing agent in the liquid also protects particles from agglomeration. The major limitation of RESS and RESOLV processes is that they are applicable only to products that show a reasonable solubility in the selected supercritical fluid. Unfortunately, many solid compounds with high molecular weight and polar bonds, that could be candidate to nanoparticles generation, show a very low or negligible solubility in $\mathrm{SC}-\mathrm{CO}_{2}$, that is the most widely SCF used and show a reduced solubility in many other compounds that could be good candidates to act as SCF. RESOLV has also the problem of the recovery of particles from the liquid solution used to improve the process performance: in this configuration, the process is no more solventless [40-43]. Turk et al. [9] used the RESS process to produce $\beta$-sitosterol (an anticholesteremic) nanoparticles of about $200 \mathrm{~nm}$ mean diameter. They tested the process in $\mathrm{SC}-\mathrm{CO}_{2}$ at different pre-expansion temperatures and pressures and observed that in the case of $\beta$-sitosterol the variation of pre-expansion conditions does not lead to appreciable variations in nanoparticles diameters. 
They also used this process for the production of griseofulvin (an antibiotic) nanoparticles using supercritical $\mathrm{CHF}_{3}$. Sane et al. [9] used RESS to produce fluorinated tetraphenylporphyrin (a photosensitizer for photodynamic therapy) spherical, agglomerated nanoparticles with average particle sizes from 60 to $80 \mathrm{~nm}$, at different pre-expansion temperatures. Pestov et al. [9] used RESS to produce nanoparticles of 2,5-distyrylpyrazine (DSP), (a polymer that can change properties upon exposure to light or chemicals, with application as optical data storage and chemical sensor). They precipitated DSP from $\mathrm{CHClF}_{2}$ and an increase of photoreactivity of nanoparticles was also observed. Sun and Rollins [9] proposed a RESOLV process in which the liquid receiving the spray of the supercritical solution also contains a reactant for the solute that nucleates from the expanding jet. They performed the rapid expansion of $\mathrm{Cd}\left(\mathrm{NO}_{3}\right)_{2}$ in $\mathrm{SC}$-ammonia into a room-temperature solution of $\mathrm{Na}_{2} \mathrm{~S}$ in water or ethanol, producing cadmium sulfide (CdS) nanoparticles with an average diameter of $3.3 \mathrm{~nm}$. The same research group [9] used the RESOLV process to produce ibuprofen (anti-inflammatory) nanoparticles with an average diameter of $40 \mathrm{~nm}$ and a particle size distribution with a standard deviation (S.D.) of $8.5 \mathrm{~nm}$. Kropf et al. [9] patented a RESS-like process in which the supercritical solution is expanded into a gas or liquid thus producing nanoparticles that can range between 10 and $300 \mathrm{~nm}$. Afurther modification of the RESOLV process consists of the use of a water in supercritical $\mathrm{CO}_{2}(\mathrm{w} / \mathrm{c})$ microemulsion used as a modified supercritical solvent to dissolve $\mathrm{AgNO}_{3}$. A w/c microemulsion containing $\mathrm{AgNO}_{3}$ was rapidly expanded into a room temperature solution of sodium borohydride. The final product of the reaction were $\mathrm{Ag}$ nanoparticles with a mean particle size of $7.8 \mathrm{~nm}$. In a subsequent work the reductive solution at the receiving end of the rapid expansion was adjusted to be highly basic. Nanocrystalline Ag particles with a bimodal distribution were obtained, with the smaller ones centered around $3.1 \mathrm{~nm}$ (S.D. $0.8 \mathrm{~nm})$ and larges ones around $10 \mathrm{~nm}$ (S.D. $2 \mathrm{~nm}$ ). The list of compounds processed down to nanometric dimensions by RESS and RESOLV is reported in Table 2. Yttrium, samarium and neodymium acetates, that are precursors of high temperature superconductors, have been micronized using dimethyl sulfoxide (DMSO) and $N$-methyl 2-pyrrolidone (NMP) as liquid solvents. 
Table2. Compounds produced in nanoparticles by RESS $[9,10]$

\begin{tabular}{llll}
\hline Material & \multicolumn{2}{l}{$\begin{array}{l}\text { Particle dimensions } \\
\text { (nm) }\end{array}$} & Note \\
\cline { 2 - 3 } & Range & Mean size & \\
\hline $\mathrm{CdS}$ & $2.5-4$ & 3.3 & RESOLV \\
$\mathrm{pbS}$ & & & RESOLV \\
$\mathrm{Ag}$ & & 7.8 & RESOLV+w/c \\
Griseofulvin & & $200 \pm 50$ & \\
$\beta$-sitosterol & & $200 \pm 50$ & \\
Fluorinated etraphenylporphyrin & & & \\
DSP & $100-300-80$ & \\
Ibuprofen & & 40 & \\
Naproxen & & 64 & RESOLV \\
PHDFDE & & 41 & RESOLV \\
Ag & & $3.1: 10$ & RESOLV \\
\hline
\end{tabular}

\subsection{SAS}

Supercritical anti-solvent precipitation (SAS) has been proposed using various acronyms; but, the process is substantially the same in all the cases. A liquid solution contains the solute to be micronized; at the process conditions, the supercritical fluid should be completely miscible with the liquid solvent; whereas, the solute should be insoluble in the SCF. Therefore, contacting the liquid solution with the SCF induces the formation of a solution, producing supersaturation and precipitation of the solute. The formation of the liquid mixture is very fast due to the enhanced mass transfer rates that characterize supercritical fluids and, as a result, nanoparticles could be produced (Fig. 2) [11, 36-44].

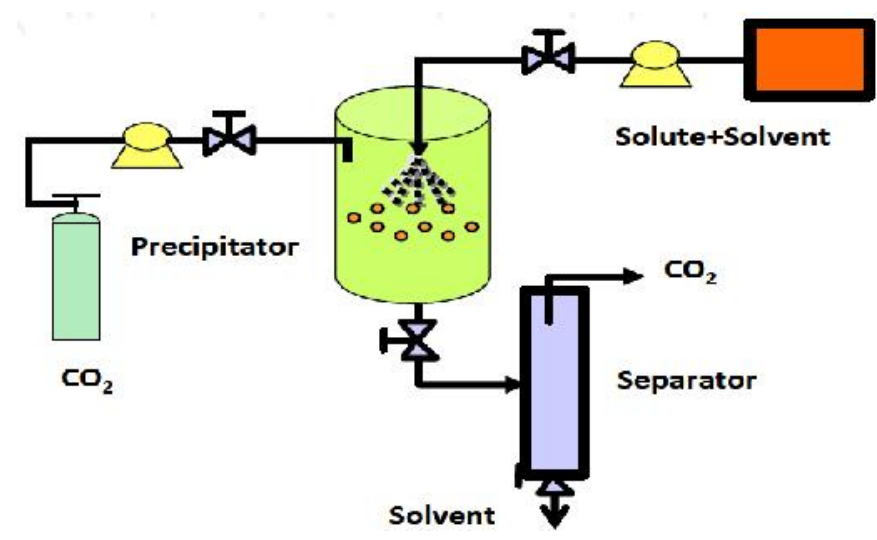

Fig.2. SAS process diagram [11] 
SAS process has been used by several authors using different process arrangements; however, the most significant differences are related to the way the process operates: in batch or semi-continuous mode. In batch operation (GAS: Gas AntiSolvent) the precipitation vessel is loaded with a given quantity of the liquid solution and, then, the supercritical antisolvent is added until the final pressure is obtained. In the semi-continuous operation (SAS), the liquid solution and the supercritical anti-solvent are continuously delivered to the precipitation vessel in co-current or counter-current mode. An important role is also played by the liquid solution injection device. The injector is designed to produce liquid jet break-up and the formation of small droplets to produce a large mass transfer surface between the liquid and the gaseous phase. Several injector configurations have been proposed in the literature and patented. High pressure vapor-liquid equilibria (VLE) and mass transfer between the liquid and the SCF also play a relevant role in SAS. Gas Anti-Solvent (GAS) process has been developed in order to achieve nanosizing of the hydrophobic materials that cannot be processed by RESS technique owing to their poor solubility in SCFs. The origin of GAS process is based on the fact that when a solution is expanded sufficiently by a gas, the liquid phase is no longer a good solvent for the solute and nucleation occurs. For example, a gaseous anti-solvent was used to reduce the lower critical solution temperature of polymer solutions to concentrate polymers $[9,11]$. Snavely et al. [9] produced insulin (antidiabetic) nanoparticles by SAS with the aid of an ultrasonic nozzle. They obtained a powder consisting of physical aggregates of $50 \mathrm{~nm}$ spheres forming sponge-like and cob-web-like structures that could be deagglomerated in smaller units. The formation of cob-web structures has also been reported by other authors and is probably due to the collision and the random coalescence of the nanometric particles. In some cases these structure collapse by sonication. Nanoparticles of some polymers have also been produced. Dextran (a bio-polymer) has been processed using DMSO. The particles produced have a spherical morphology and a mean particle size ranging between 125 and $150 \mathrm{~nm}$ [9]. Pilot scale SAS experiments confirmed these results [9] and the possibility to scale-up SAS process. Fig. 3 reports a SEM image of dextran nanoparticles produced using the pilot scale SAS apparatus located at the University of Salerno (Italy). At University of Cadiz, ethyl cellulose microparticles were successfully precipitated from 
dichloromethane (DCM) by SAS process [11] and particles were reduced from 50-100 to 3-5 m. The concentration was the factor that had the greatest influence on the PS and PSD. An increase in the initial concentration of the solution led to larger particles sizes with a wider distribution. The list of compounds processed by SAS down to nanometric dimensions is reported in Table 3.

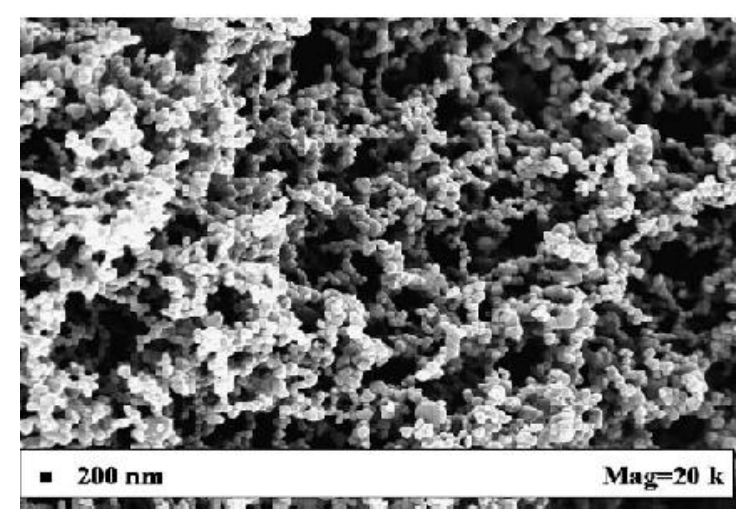

Fig.3. SAS precipitated dextran nanoparticles, from DMSO, $10 \mathrm{mg} / \mathrm{mL}, 150$ bar, $40 \propto \mathrm{C}$ (pilot plant) [9]

Table3. Compounds produced in nanoparticles by SAS [9]

\begin{tabular}{llll}
\hline Material & \multicolumn{2}{l}{$\begin{array}{l}\text { Particle dimensions } \\
(\mathrm{nm})\end{array}$} & Note \\
\cline { 2 - 3 } & Range & Mean size & \\
\hline AcY & $50-150$ & 100 & RESOLV \\
AcSm & $80-120$ & $\sim 100$ & RESOLV \\
AcZn & $<30$ & 50 & RESOLV+w/c \\
Tetracycline & & 150 & \\
HPMA & & 150 & \\
Dextran & & $125-150$ & \\
Fullerene & & $29-83$ & SAS batch \\
Griseofulvin & & 130 & SAS-EM (GAS) \\
Lysozyme & & 190 & SAS-EM (GAS) \\
Lysozyme & & 180 & GAS \\
Insulin & & 50 & \\
PLLA & & $193 \pm 20$ & \\
Pigment Red 177 & & $49-74$ & \\
Disperse Red 60 & $50-100$ & \\
& & &
\end{tabular}




\subsection{SAA}

Supercritical assisted atomization (SAA) is a recent process in which the SCF acts as atomizing medium. The process is based on the solubilization of supercritical $\mathrm{CO}_{2}$ in the liquid solution formed by the solvent and the (solid) solute, and on its subsequent atomization using a thin wall nozzle. When SAA is properly conducted, two atomization processes take place: the first one is the production of primary droplets at the exit of the nozzle by pneumatic atomization; the second one destroys these droplets by the fast release of $\mathrm{CO}_{2}$ from the internal of the droplet (decompressive atomization). Amorphous or crystalline particles have been produced, depending on the process temperatures and the chemical characteristics of the solid solute $[9$, 41]. Using SAA, PMMA (polymethylmethacrylate) nanoparticles have been obtained using acetone as solvent. At the concentration of $10 \mathrm{mg} / \mathrm{mL}$ of PMMA in acetone and at a mixing temperature of $80{ }^{\circ} \mathrm{C}$ and a mixing pressure of 76 bar, particles with a mean diameter of 120 nm have been produced [9]. The use of SCFs is well established in gels drying since it allows the drying process with zero surface tension, avoiding the gel collapse. In this process, the SCF is only used to recover the produced nanoparticles (Table 4).

Table4. Compounds produced in nanoparticles by SC-drying [9]

\begin{tabular}{lll}
\hline Material & Particle dimensions (nm) & Note \\
\hline $\mathrm{Cu}_{3} \mathrm{~B}_{2} \mathrm{O}_{6}$ & $10-20$ & SC-CO \\
$\mathrm{TiO}_{2}$ & $10-20$ & SC-CO \\
$\mathrm{Be}_{2} \mathrm{BO}_{3}(\mathrm{OH})$ & 7 & SC-CO \\
$\mathrm{Mg}_{2} \mathrm{~B}_{2} \mathrm{O}_{5}-\mathrm{MgB}_{6} \mathrm{O}_{10}$ & 10 & SC-EtOH \\
$5 \mathrm{H}_{2} \mathrm{O}-\mathrm{MgB}_{2}$ & & \\
\hline
\end{tabular}

\subsection{Hydrothermal synthesis in supercritical water}

Hydrothermal synthesis (HTS) is used to produce synthetic materials imitating natural geothermal processes. The reaction equilibrium of metal salt aqueous solutions changes with temperature and results in the formation of metal hydroxides or metal oxides. Supercritical water (SCW) provides an excellent reaction medium for hydrothermal synthesis, since it allows varying the reaction rate and equilibrium by shifting the dielectric constant and solvent density with pressure and temperature. One of the expected benefits is higher reaction rates 
and smaller particles. The reaction products. The HTS-SCW process is usually operated as follows: a metal salt aqueous solution is prepared, pressurized and heated. The pressurized metal salt solution and a supercritical water stream are combined in a mixing point, which leads to rapid heating and subsequent reaction. After the solution leaves the reactor, it is rapidly quenched and in-line filters remove larger particles. Cooling water is directly fed to the reactor to quench the reaction (Fig. 4). Two different process modes have been proposed: the first uses a batch reactor and is characterized by a long reaction time; the second uses a flow reactor that assures continuous operation [10,44].

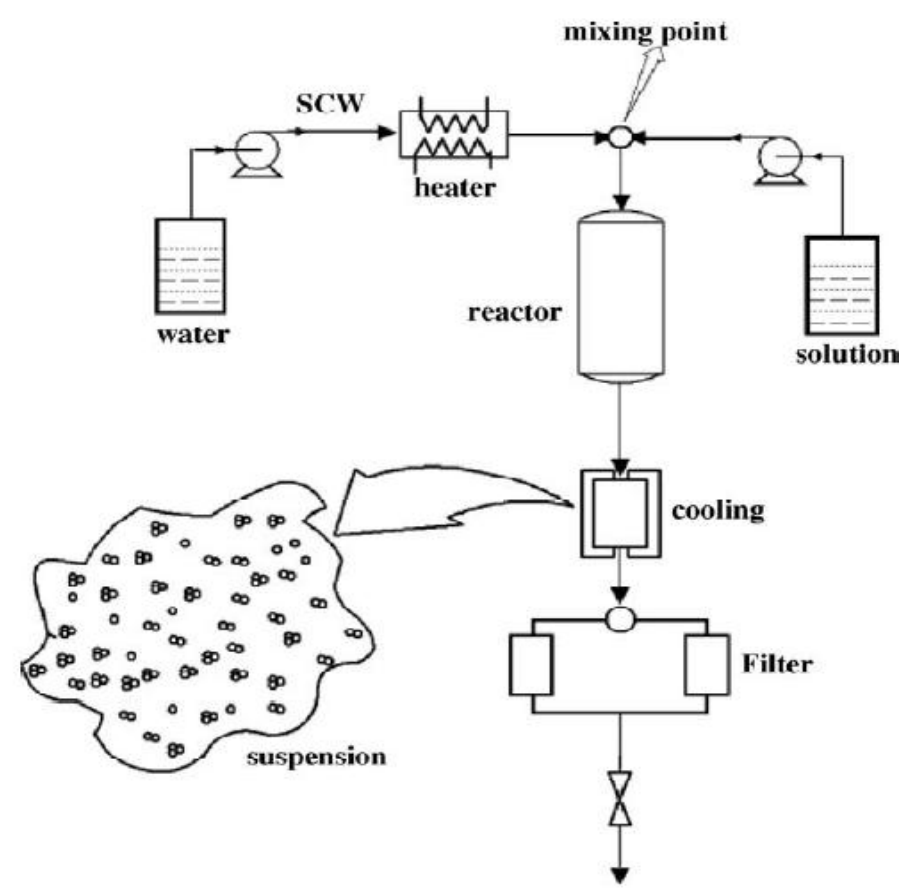

Fig.4. A schematic representation of the HTS-SCW process [9]

$\mathrm{Hu}$ et al. [9] proposed the preparation of gels in aqueous solution, the replacement of water in the precipitate with a mixture of n-propanol and benzene and, then, the elimination of the organic solution using $\mathrm{SC}-\mathrm{CO}_{2}$. In this case the process was finalized to the production of copper borate nanoparticles. $\mathrm{Hu}$ et al. [9] proposed the formation of $\mathrm{TiO}_{2}$ nanoparticles by sol preparation and the replacement of water in the precipitate with n-butanol and subsequent supercritical drying. HTS-SCW process has been extensively explored by Adshiri et al., Hakuta et al., and $\mathrm{Li}$ et al. [45] in various works to produce nanometric particles of several single and complex metal oxides. This research groups used several types of flow apparatus to 
perform HTS-SCW experiments. They evidenced several parameters of the process that can control dimension and morphology of the produced nanoparticles. First of all, solubility of metallic oxides in SCW has to be considered and its dependence on temperature and $\mathrm{pH}$. Also the initial concentration of the feed and the heating rate can play a relevant role in this process. Caba nas et al. [10] used a small continuous reactor to produce $\mathrm{Ce} 1-{ }_{x} \mathrm{Zn}_{x} \mathrm{O}_{2}$ by HTS-SCW starting from mixtures of cerium ammonium nitrate and zirconium acetate. According to these authors, the continuous reactor allows a better control of the experimental conditions when compared to the batch process. Very small nanoparticles have been obtained. After calcination at $1000{ }^{\circ} \mathrm{C}$ for $1 \mathrm{~h}$ the material sintered up to $145 \mathrm{~nm}$. In a subsequent work [10] they used continuous HTSSCW to obtain nanoparticles of $\mathrm{Fe}_{3} \mathrm{O}_{4}$ and various $\mathrm{Fe}-\mathrm{Ni}-\mathrm{Zn}$ mixtures. In all cases, crystalline particles with mean diameters lower than $100 \mathrm{~nm}$ were obtained. Carbon nanotubes $(\mathrm{CNT})$ have attracted very great attention due to their structural, mechanical and electronic properties and the related technological applications. They have potential applications in semiconductor devices, field emitters, scanning probe microscopes, quantum wires, hydrogen and other gases storage devices. Other possible uses include materials for batteries and fuel cells, capacitors and chemical filters [9]. Several methods of CNT preparation have been proposed and can be classified in physical and chemical methods. Chemical methods seem to be more efficient in terms of yield and energy consumption, but, can produce lower quality carbon nanotubes. Carbon nanotubes production has also been proposed using SCF based processes. Calderon-Moreno et al. [10] reported a HTS-SCW process to produce CNTs from amorphous carbon; no catalysts were used. Lee et al. [46] proposed the synthesis of CNTs in SC toluene; catalysts like Fe or Ferrocene were also used. In this process toluene serves as both the carbon source for nanotubes and as the solvent for the reaction. Initially researchers studied single nanoparticles because such particles have much better properties than the bulk materials. Later, in the late 1980s, researchers found that heterogeneous, composite or sandwich colloidal semiconductor particles have better efficiency than their corresponding single particles; in some cases they even develop some new properties [47-50]. More recently during the early 1990s, researchers synthesized concentric multilayer semiconductor nanoparticles with the view to improving the property of 
such semiconductor materials. Hence subsequently the terminology "core/shell” was adopted. Nanoparticles can be categorized based on single or multiple materials into simple and core/shell or composite nanoparticles. In general, simple nanoparticles are made from a single material; whereas, as the name implies, composite and core/shell particles are composed of two or more materials. The core/shell type nanoparticles can be broadly defined as comprising a core (inner material) and a shell (outer layer material). These can consist of a wide range of different combinations in close interaction, including inorganic/inorganic, inorganic/organic, organic/inorganic, and organic/organic materials. The choice of shell material of the core/shell nanoparticle is generally strongly dependent on the end application and use [50]. Garriga et al. [51] presented a new way for particle coating. It consists of depositing on a core particle a thin layer of a material based on copper in a supercritical medium. Two examples are described: a deposit of $\mathrm{Cu}$ on nickel particles and a deposit of $\mathrm{Cu}$ on particles of permanent magnet $\mathrm{SmCo}_{5}$. In both cases the copper source was bis(hexafluoroacetylacetonate)copper(II), which is thermally decomposed in the supercritical mixture $\mathrm{CO}_{2}$ /ethanol. This process allows one to obtain new "core-shell structures", $\mathrm{Ni} / \mathrm{Cu}$ and $\mathrm{SmCo}_{5} / \mathrm{Cu}$. New interesting properties are expected for these structures, more particularly in the magnetic recording field. The strong point of their process was its capability to coat spherical particles in order to permit the compatibility of the core material for its further processability. Results exhibit the coating influence on the magnetic properties of the core materials. In the case of $\mathrm{Ni}$ particles, the coating allows one to isolate the magnetic particles and to increase the coercive field. Now the work is focused on the $\mathrm{SmCo}_{5}$ particles in order to develop new improved materials for magnetic recording systems (Fig. 5). Pradeep et al. [52] synthesized $\mathrm{CeO}_{2}-\mathrm{ZrO}_{2}$ composite nanoparticles with mesoporous and spherical morphologies were assembled into different homogeneously dispersed, domain, and core-shell structures by changing the reaction conditions, such as the heating rate, acid, and solvent, in supercritical alcohols (methanol and 2-propanol). They represented which the choice of the solvent played a key role in controlling the thickness of the outer shell. Methanol gave a very thin outer shell of cubic $\mathrm{CeO}_{2}$, while a thick shell formed when 2-propanol was used. Pradeep et al. succeeded in controlling the structures and morphologies of mesoporous spherical $\mathrm{CeO}_{2}-\mathrm{ZrO}_{2}$ 
NPs with an easy synthetic method.
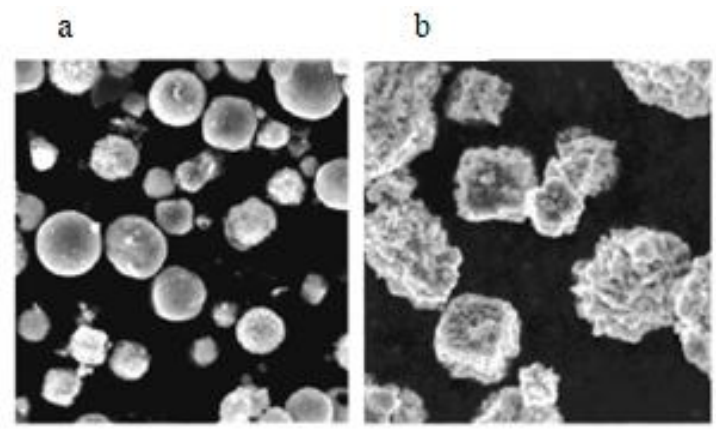

c

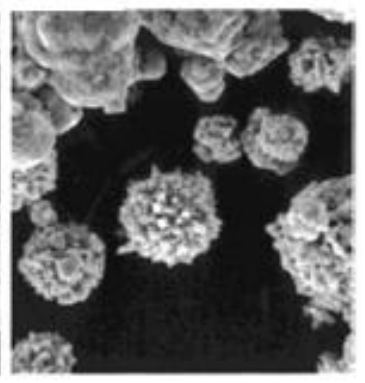

Fig.5. SEM pictures of Ni particles: (a) initial; (b) after the supercritical treatment, with $\mathrm{T}=473 \mathrm{~K}$; (c) after coating, with $\mathrm{T}=473 \mathrm{~K}$. [51]

\section{CONCLUSION}

A large quantity of SCF based processes that were successful in producing nanomaterials has been found in the literature at laboratory scale. They can be divided in new processes and adaptation of existing processes to the use of SCFs. In all cases, using SCFs, more flexible and/or simplified processes have produced and with a reduction of the environmental impact. The final result is that nanomaterials with potentially better performances have been obtained using improved processes. Probably not all the SCF based processes that have been proposed will find an application; a "natural" selection is expected among them and only some will be used. The application to a large variety of nanomaterials has been proposed and we can expect that different materials will be tested in the future. RESS and SAS processes have been applied on the particle precipitation and co-precipitation of many active ingredient/polymer. Furthermore, SAS has advantages over RESS because SAS is usually operated under mild conditions compared with those of RESS, which is associated to relatively high temperature and high pressure. Anyway, the technical viability of the SAS process requires knowledge of the phase equilibrium of the system; its hydrodynamics, the mass transfer between the jet generated and the continuous phase; and the mechanisms and kinetics of nucleation and crystal growth.

\section{REFERENCES}

[1] Yoon S. H., Yang H., Kim Y. S., "Ordered growth of $\mathrm{ZnO}$ nanorods for fabrication of a hybrid plasma display panel", Nanotechnology, 2007, 18, 205-608. 
[2] Kim H. J., Lee C. H., Kim D. W., Yi G. C., Fabrication and electrical characteristics of dual-gate $\mathrm{ZnO}$ nanorod metal-oxide semiconductor field-effect transistors. Nanotechnology, 2006, 17. S327-S331.

[3] www.nano.ir

[4] Priyanto S., Mansoori A. G., Suwono A. Measurement of property relationships of nano-structure micelles and coacervates of asphaltene in a pure solvent. Chemical Engineering Science, 2001, 56, 6933-6939.

[5] Tillotson TM., Gash AE., Simpson RL., Hrubesh LW., Satcher JH., Poco JF. Nanostructured energetic materials using sol-gel methodologies. J. Non-Cryst Solids. 2001, 285. 338-45.

[6] Lu AH., Salabas EL., Schuth F. Magnetic nanoparticles: synthesis, protection, functionalization, and application. Angew Chem Int Ed. 2007, 44-46.

[7] Zhao L., Zhang H., Tang J., Song S., Cao F. Fabrication and characterization of uniform $\mathrm{Fe}_{3} \mathrm{O}_{4}$ octahedral micro-crystals. Mater Lett.2009, 63. 307-314.

[8] Roco M.C., The long view of nanotechnology development: The National Nanotechnology Initiative at 10 years. In Nanotechnology research directions for societal needs in 2020 (pp. 1-28). Amsterdam: Springer Netherlands. 2011.

[9] Reverchon E., Adami R. Nanomaterials and supercritical fluids. J. of Supercritical Fluids, 2006, 37, 1-22.

[10] Byrappa K., Ohara S., Adschiri T. Nanoparticles synthesis using supercritical fluid technology - towards biomedical applications, 2008, 60, 299-327.

[11] Montes A., Gordillo M. D., Pereyra C., Martinez E. J. Particles Formation Using Supercritical Fluids. Mass Transfer - Advanced Aspects, 2011.

[12] Ollanketo M., Hartonen K., Riekkola M., Holm Y., Hiltunen R. Supercritical carbon dioxide extraction of lycopene in tomato skins, Eur. Food Res. Technol. 2001, 561-565.

[13] Saito M. History of supercritical fluid chromatography: Instrumental development. J. Bioengineering. 2013, 115(6), 590-599.

[14] Smith R. M. Supercritical fluid in separation sciencedthe dreams, the reality and the future J. Chromatogr. A, 1999, 856, 83-115. 


\section{[15] http://webbook.nist.gov/}

[16] Matsubara A., Fukusaki E., Bamba T.. Metabolite analysis by supercritical fluid chromatography. Bioanalysis, 2010, 2, 27-34.

[17] Pinkston J. D., Stanton D. T., Wen D. Elution and preliminary structureretention modeling of polar and ionic substances in supercritical fluid chromatography using volatile ammonium salts as mobile phase additives, J. Sep. Sci., 2004, 27, 115-123.

[18] Li F., Hsieh Y. Supercritical fluid chromatography-mass spectrometry for chemical analysis. J. Sep. Sci., 2008, 31, 1231-1237.

[19] Yamini Y., Bahramifar N. Solubility of polycyclic aromatic hydrocarbons in supercritical carbon dioxide. J. Chem. Eng. Data, 2000, 45, 53-56.

[20] Housaindokht M. R., Bozorgmehr M. R. Calculation of solubility of methi- mazole, phenazopyridine and propranolol in supercritical carbon dioxide J. Supercrit. Fluids, 2008, 43, 390-397.

[21] Knez Z., Markocic E., Leitgeb M., Primozic M., Hrncic M. K., Skerget M. Industrial applications of supercritical fluids: A review. Energy, 2014, 77, 235-243.

[22] Jessop PG., Leitner W. Chemical synthesis using supercritical fluids. Weinheim: Wiley-VCH; 1999.

[23] Ramsey E., Sun Q., Zhang Z., Zhang C., Gou W. Mini-review: green sustainable processes using supercritical fluid carbon dioxide. J. Environ Sci, 2009, 21,720-6.

[24] Sovilj MN., Nikolovski BG., Spasojevic MD. Critical review of supercritical fluid extraction of selected spice plant materials. Maced J Chem Chem Eng, 2011, 30, 197-220.

[25] Yang H., Zhong C. Modeling of the solubility of aromatic compounds in supercritical carbon dioxide-cosolvent systems using SAFT equation of state J. of Supercritical Fluids, 2005, 33, 99-106.

[26] Munshi P., Bhaduri S. Supercritical $\mathrm{CO}_{2}$ : a twenty-first century solvent for the chemical industry. Curr Sci India, 2009, 97, 63-72.

[27] Dong Z., Li Y., Lin M., Li M. A study of the mechanism of enhancing oil recovery using supercritical carbon dioxide microemulsions. Pet Sci, 2013, 10, 91-96.

[28] Cooper AI. Polymer synthesis and processing using supercritical carbon dioxide. J. Mater 
Chem, 10, 207-214. 2000.

[29] Markocic E., Kramberger B., Bennekom JG. V., Heeres H. J., Vos J., Knez Z. Glycerol reforming in supercritical water; a short review. Renew Sust Energ Rev, 8, 23-40. 2013.

[30] Wang T. Ch., Chang P. Ch. Measurement and Correlation for the Solid Solubility of Antioxidants Sodium L-Ascorbate and Sodium Erythorbate Monohydrate in Supercritical Carbon Dioxide. J. Chem. Eng. Data, 60, 790-794. 2015.

[31] Habulin M., Primozic M., Knez Z. Supercritical fluids as solvents for enzymatic reactions. Acta Chim Slov, 54, 667-677. 2007.

[32] Skerget M., Knez Z., Hrncic M. K. Solubility of Solids in Sub- and Supercritical Fluids: a Review. J. Chem. Eng. Data, 56, 694-719. 2011.

[33] Knez Z., Weidner E. Particles formation and particle design using supercritical fluids. Curr Opin Solid State Mater, 7, 353-361. 2003.

[34] McHugh M. A., Krukonis V. J. Supercritical fluid extraction: principles and practice. The University of Michigan: Butterworth-Heinemann. 1994.

[35] Cabanas A., Poliakoff M. The continuous hydrothermal synthesis of nano-particulate ferrites in near critical and supercritical water. J. of Mater Chem 11. 1408-1416. 2001.

[36] Lee S. M.,. Bae Y. Ch. Swelling Behaviors of Doubly Thermosensitive Core-Shell Nanoparticle Gels. Macromolecules 47, 8394-8403, 2014.

[37] Adschiri T, Kanazawa K, Arai K. Rapid and Continuous Hydrothermal Synthesis of Boehmite Particles in Subcritical and Supercritical Water. J.Am.Ceram.Soc, 1992, 75. 261518.

[38] Hakuta Y., Adschiri T., Suzuki T., Chida T., Seino K., Arai K. Flow method for rapidly producing barium hexaferrite particles in supercritical water. J. of Am. Ceram. Soc., 81. 24612464. 1998.

[39] Hakuta Y., Onai S., Adschiri T., Arai K. Production of ultra-fine ceria particles by hydrothermal synthesis under supercritical conditions. J. Mat. Sci. Lett., 1998, 17. 1211-13. [40] Jung J., Perrut M. Particle design using supercritical fluids: literature and patent survey. J. Supercrit. Fluids 20. 179-219. 2001.

[41] Adschiri T., Hakuta Y., Arai K. Hydrothermal synthesis of metal oxide fine particles at 
supercritical conditions. Ind. Eng. Chem. Res., 39. 4901-4907. 2000.

[42] Hu Z. S., Dong J. X., Chaen G. X., Lou F. Preparation of nanometer copper borate with supercritical carbon dioxide drying”, Poweder Technol. 165-171. 1999.

[43] Reverchon E., Antonacci A. Polymer microparticles production by supercritical assisted atomization", J. of supercritical Fluids 39. 444-452. 2007.

[44] Reverchon E. Supercritical-assisted atomization to produce micro and/or nanoparticles of controlled size and distribution”, Ind. Eng. Chem. Res. 41. 24-35. 2005.

[45] Choi H., Veriansyah B., Kim J., Kim J. D., Kang JW. Continuous synthesis of metal nanoparticles in supercritical methanol. J. of Supercrit Fluids. 52. 285-291. 2010.

[46] Kim J., Kim D., Veriansyah B., Kang JW., Kim J. D. Metal nanoparticle synthesis using supercritical alcohol”. Mater Lett. 63. 1880-1889. 2009.

[47] Ruohong S., Charpentier P. Synthesis of Metal Oxide Nanostructures by Direct Sol-Gel Chemistry in Supercritical Fluids", Journal of Chem. Rev, 112, 3057-3082. 2012.

[48] Veriansyah B., Kim J-D., Min B. K., Kim J. Continuous synthesis of magnetite nanoparticles in supercritical methanol. Materials Letters, 64, 2197-2200. 2010.

[49] Choia H., Veriansyah B., Kim J., Kim J-D., Kang J. W. Continuous synthesis of metal nanoparticles in supercritical methanol” J. of Supercritical Fluids, 52, 285-291, 2010.

[50] Chaudhuri R. G., Paria S. Core/Shell Nanoparticles: Classes, Properties, Synthesis Mechanisms, Characterization, and Applications”, Chem. Rev, 112, 2373-2433. 2012.

[51] Pessey V., Garriga R., Weill F., Chevalier B., Etourneau J., Cansell F. Core-Shell Materials Elaboration in Supercritical Mixture $\mathrm{CO}_{2} /$ Ethanol”, Ind. Eng. Chem. Res. 39, 4714-4719. 2000.

[52] Pradeep E. K. C., Habu T., H. Tooriyama, M. Ohtani, K. Kobiro, "Ultra-simple synthetic approach to the fabrication of $\mathrm{CeO}_{2}-\mathrm{ZrO}_{2}$ mixed nanoparticles into homogeneous, domain, and core-shellstructures in mesoporous spherical morphologies using supercriticalalcohols", J. of Supercritical Fluids. 97, 217-223. 2015.

\section{How to cite this article:}

Bagheri Hamidreza A, Soltani Reza B, Shahmirzaei Mahdi C, Yahyanejad Mahdi D, Fijani Fatemeh E, Hashemipour Hassan F. Nanotechnology and supercritical fluids. J. Fundam. Appl. Sci., 2016, 8(2S), 839-859. 